\title{
The Local Bubble in the interstellar medium and the origin of the low energy cosmic rays
}

\author{
${ }^{*}$ A.D. Erlykin ${ }^{1,2}$, S.K. Machavariani ${ }^{1}$, A.W. Wolfendale ${ }^{2}$ \\ (1) P N Lebedev Physical Institute, Moscow 119991, Russia \\ (2) Physics Department, Durham University, Durham, DH1 3LE, UK
}

June 23, 2021

\begin{abstract}
An analysis of the energy spectra of cosmic rays and particularly the precise data from the AMS-02 experiment support the view about the important role of the Local Bubble in the nearby interstellar medium. It is suggested that the bulk of CR below about $200 \mathrm{GV}$ of rigidity (momentum/charge ratio) comes from the modest number of supernova remnants in the Local Bubble which appear to have occurred some $10^{6}$ years ago and contributed to its formation. At higher rigidities the contribution from a 'Local Source', a single supernova remnant generated some $10^{5}$ years ago seems to dominate up to, at least $1000 \mathrm{GV}$.
\end{abstract}

Keywords: cosmic rays, local bubble, energy spectrum

\section{Introduction}

The origin of cosmic rays (CR) is still subject of some doubt, although supernova remnants (SNR) appear to play an important part, at least to several PV (Ginzburg and Syrovatskii, 1964 and many other publications). Recent precise measurements of the rigidity spectra of protons and helium nuclei, the positron fraction, the antiproton to proton ratio and preliminary data on the rigidity spectrum of lithium and boron to carbon ratio (Ting for AMS-02 coll., 2015) all support the previous view (Erlykin and Wolfendale, 2013, 2015a,b; Tomassetti, 2015a,b; Kachelriess et al., 2015) that there is a large contribution to the intensity from a nearby, recent SNR above $200 \mathrm{GV}$, particularly for the secondary nuclei (Erlykin and Wolfendale, 2016a; Tomassetti, 2015a,b).

We turn now to rigidities below $200 \mathrm{GV}$ where earlier we proposed that a 'New Component' dominates (Erlykin and Wolfendale, 2012). This idea has been put forward

\footnotetext{
${ }^{1 *}$ Corresponding author: tel +74991358737

E-mail addresses: erlykin@sci.lebedev.ru; machavar@sci.lebedev.ru; a.w.wolfendale@durham.ac.uk
} 
more recently in (Tomassetti, 2015c,d) for, at least, carbon and oxygen. Thus, if the above is correct, the idea of $\mathrm{CR}$ at Earth being largely derived from very many SNR in the Galaxy needs revision.

Here we put forward the idea that the origin scenario is more complicated, with significant features due to many, say, tens of SNR (analogous to multiple scattering of particles), a modest, say, several number of SNR, locally (analogous to plural scattering) and a single SNR (analogous to a single Rutherford scattering). Here, the analogy with the classification of scatterings is given simply as a visual illustration. In the present paper we deal with the 'modest number of SNR, locally'. This involves an examination of the local environment of the Solar System in the Local Bubble, a region in which low energy CR isotopes have been hypothesised to be accelerated (Binns et al., 2007, 2016).

Experimental study of the CR isotope composition may provide information on the matter content and diffusion properties of the local ISM, but it is not easy because of the low density of such isotopes. It is particularly true for radioactive isotopes, such as ${ }^{10} \mathrm{Be},{ }^{26} \mathrm{Al},{ }^{60} \mathrm{Fe}$ and others, the low density of which can be due not only to low density within the Local Bubble, but by their decay too. The problem has been discussed in many papers, for example in Ptuskin and Soutoul (1998).

\section{The Local Bubble}

There is no doubt that the Solar System currently lies in a Local Bubble of effective radius 200pc (Frisch, 1997). The Local Bubble contains hot gas of low density $\left(10^{6} \mathrm{~K}\right.$; $10^{-2} \mathrm{~cm}^{-3}$ ) (Frisch, 1997; Berghofer and Breitschwerdt, 2002) and was probably caused by a succession of supernovae (about 10) starting some $10^{6}$ years ago and by associated stellar winds from massive stars. Such a number of SNe can be compared with that expected on the basis of the usually assumed galactic frequency of $10^{-2}$ year $^{-1}$, an effective galactic radius of $15 \mathrm{kpc}$ and a disk thickness of $0.3 \mathrm{kpc}$, leading to an expected number of 2 for a uniform SNR distribution in space and time. Thus, the actual number of local SNR is a little smaller than expected.

It must be realised, however, that the actual volume in which the supernova formed would have been much smaller than the 'radius 200 pc'. This is because the relevant OB Associations have radii of only about 20pc (Mel'nik and Efremov, 1995) and the actual number of 'local SNR' could have exceeded the number expected by a large factor $(\approx 100)$. What is certain is that the Solar System is in an unusually hot interstellar medium over a period of some $10^{6}$ years where there has been 'recent' supernova activity.

\section{The expected cosmic rays from the supernovae in the Local Bubble}

In Erlykin and Wolfendale, (2015a) we evaluated the proton spectra expected from Geminga and Monogem SNR and compared them with our estimate of the 'observed' spectrum from a single source, using the AMS-02 results. The latter was derived from 
the measured proton spectrum minus the assumed 'background'. Since this 'background' was defined as that due to CR produced by many old and distant SNR contributing mostly in the low energy region we fitted its spectrum in this region by its power law and extrapolated it to energies higher than that of the kink at $300 \mathrm{GV}$ (Erlykin and Wolfendale, 2015a, Figure 2). The calculations of the spectra from Geminga and Monogem SNR were made for the situations of alternatively normal and anomalous diffusion. The anomalous diffusion is more appropriate for the non-uniform ISM with bubbles and variety of densities and temperatures. We expect the diffusion to be anomalous for the conditions inside the Local Bubble and for some distance beyond (Erlykin et al., 2003). The result was a prediction lower than 'observed' by a factor 30 at $1000 \mathrm{GeV}$ for Geminga and anomalous diffusion. Three factors combine to account for the intensity of the New Component:

(a) The fact, referred to in section 2, that there has been a succession of supernovae locally over the past $10^{6}$ to $10^{7}$ years.

(b) The increased temperature which would have started after the first few supernovae and would have caused a considerable increase in the CR injection efficiency (Erlykin et al., 2016a).

(c) The existence of a Bubble implies the trapping of particles and plasma within. The Cygnus Bubble is an example (eg. Ackermann et al., 2011) and the Fermi Bubble near the Galactic Centre (Ackermann et al., 2014) is another. Indeed, the spectral shape of the excess from the Fermi Bubble is similar to what we expect from our own New Component. The fact that the Galactic Explosion at the Galactic Centre some 5My ago gives a Bubble that is still visible - with its sharp edges - is in the spirit of the situation for our Local Bubble. It should be added that Higdon et al. (1998) stressed the role of Cosmic Ray acceleration from SN ejecta in Superbubbles nearly 20 years ago.

Reiterating (c) the problem is in dissuading the new CR from leaving the local interstellar medium; under normal conditions the CR generated some few million years ago would have diffused of several kpc by now. In this respect the Local Bubble can be invoked as a trapping volume.

The increased injection efficiency with increased interstellar medium temperature is important (point (b)). In our work (Erlykin et al., 2016b) we explained the remarkably small CR intensity gradient in the Galaxy in terms of the radial gradient of the interstellar medium temperature. The injection efficiency was shown to increase very rapidly with increasing temperature. The result of relevance here is that the efficiency in the Local Bubble should be very high, the later supernovae benefitting from the increased interstellar medium temperature arising from the earlier ones. Extrapolating the plots of the fraction of ambient protons of energy sufficient to be injected for CR acceleration as a function of temperature yields efficiencies approaching $100 \%$ for the very high Local Bubble temperature (Erlykin et al., 2016b, Figure 4).

It is postulated that it is not the 'current' single source which is important at 1000 GV, that is responsible for the CR below 200 GV ('the New Component') but rather the other old SNR which generated the Local Bubble. The antiquity of the SNR means that their contributions to the CR intensity will be biased towards lower energies, the higher energy particles having left the Local Bubble. Lack of knowledge of the supernovae in 
excess and their ages coupled with uncertainty in the actual trapping efficiency of the Local Bubble precludes accurate calculations of the CR spectra at present.

The high rate of recent SNR will also mean that the 're-acceleration' process is relevant, see, for example, Thoudam and Hörandel, (2014). The weak shocks associated with old SNR will also give significant extra fluxes of CR, mainly below $200 \mathrm{GV}$ due to their larger volume. These particles will form part of the new component.

\section{Conclusion}

With data on the characteristics of the Local Bubble and the local interstellar medium, and our position within it, it is likely that a significant fraction of CR of rigidity 200 GV, and below (our 'New Component') comes from the claimed excess of supernovae responsible for the Bubble, in the last a few $10^{6}$ years.

\section{Acknowledgements}

We are grateful to the Kohn Foundation for financial support.

\section{References}

Ackermann, M., Ajello, M., Allafort, A. et al., 2011, A cocoon of freshly accelerated cosmic rays detected by Fermi in the Cygnus Superbubble, Science, 334, 1103-1107 Ackermann, M., Albert, A., Atwood, W.B. et al., 2014, The spectrum and morphology of the Fermi bubbles, 793:64(34pp)

Berghofer, T.W. and Breitschwerdt, D., 2002, The origin of the young stellar population in the solar neighbourhood - a link to the formation of the Local Bubble, Astron. and Astrophys., 390, 299-306

Binns, W.R., Wiedenbeck, M.E., Arnould, M. et al., 2007, OB Associations, Wolf-Rayet stars and the Origin of Galactic Cosmic Rays, Space Science Rev. 130, 439-449

Binns, W.R., Israel, M.H., Christian, A.C. et al., 2016, Observation of the ${ }^{60} \mathrm{Fe}$ nucleosynthesis-clock isotope in galactic cosmic rays, Science, 352, 677-680

Erlykin, A.D. and Wolfendale, A.W., 2001, Supernova remnants and the origin of the cosmic radiation I. SNR acceleration models and their predictions, J. Phys. G: Nucl. Part. Phys., 27, 941-958

Erlykin, A.D., Lagutin, A.A. and Wolfendale, A.W., 2003, Properties of the interstellar medium and the propagation of cosmic rays in the Galaxy, Astropart. Phys., 19/3, 351-362

Erlykin, A.D. and Wolfendale, A.W., 2012, A 'New Component' of cosmic rays, Astropart. Phys., 35, 449-456

Erlykin, A.D. and Wolfendale, A.W., 2013, Cosmic ray positrons from a local, middle-aged supernova remnant, Astropart. Phys., 49, 23-27

Erlykin, A.D. and Wolfendale, A.W., 2015a, Interpretation of the cosmic ray protons and helium nuclei energy spectra in terms of a local source, J. Phys. G: Nucl. Part. Phys. 42(12), 125201-125208(8pp) 
Erlykin, A.D. and Wolfendale, A.W., 2015b, Cosmic Ray antiprotons and the Single Source Model, J. Phys.G: Nucl. Part. Phys.,42(11), 115202-115207

Erlykin, A.D. and Wolfendale, A.W., 2016a, Secondary cosmic ray nuclei in the light of the Single Source model, J. Phys.G: Nucl. Part. Phys. 43/10, 105201(8pp) Erlykin, A.D., Wolfendale A.W. and Dogiel, V.A., 2016b, The radial gradient of cosmic ray intensity in the Galaxy, Adv. Space Res., 57, 519-525

Higdon, J.C., Lingenfelter, R.E., Ramaty, R., 1998, Cosmic rays accelerated from supernova ejecta in superbubbles, Astrophys. J. Lett., 509, L33-L36

Frisch, P.C., 1997, The local bubble, local fluff and heliosphere, 'The Local Bubble and Beyond' Lyman-Spitzer Colloquium, 506, 269-278; http://dx.doi.org/10.1007/BFb0104732]; arxiv:astro-ph/971014

Ginzburg,V.L. and Syrovatskii, S.I., 1964, Origin of Cosmic Rays, Pergamon Press

Kachelriess, M., Neronov, A. and Semicoz, D.V., 2015, , Signatures of a two million year old supernova in the spectra of cosmic ray protons, antiprotons and positrons Phys. Rev. Lett., 115, 181103(5pp)

Mel'nik, A M and Efremov, Yu N, 1995, A New List of OB Associations in our Galaxy, Astron. Lett., 21, 10-26

Ptuskin, V.S. and Soutoul, A., 1998, Decaying cosmic ray nuclei in the local interstellar medium, Astron. and Astrophys., 327, 859-866

Thoudam, S. and Hörandel, J.R., 2015, GeV-TeV cosmic ray spectral anomaly as due to re-acceleration by weak shocks in the Galaxy, Astron. and Astrophys., 567, A33; [http://dx.doi.org/10.1051/0004-6361/201322996]; arXiv:1404.3630

Ting, S. for the AMS-02 coll.,2015, Introduction to the AMS-02 experiment, AMS02 Days at CERN, |http://home.cern/scientists/update/2015/04/ams-days-cern Tomassetti, N., 2015a, Inhomogeneous diffusion model for recent data on high energy cosmic rays, Progress of Science, PoS(ICRC2015) 551

Tomassetti, N. and Donato, F., 2015b, The connection between the positron anomaly and the spectral features in galactic cosmic ray hadrons, Astrophys. J. Lett., 803, L15; http://dx.doi.org/10.1088/2041-8205/803/2/L15

Tomassetti, N., 2015c, Consistent description of leptonic and hadronic spectra in cosmic rays, Progress of Science, PoS(ICRC2015) 552

Tomassetti, N., 2015d, Cosmic-ray protons, nuclei, electrons and antiparticles under two-halo scenario of diffusive propagation, Phys. Rev. D 92, 081301(R); http://dx.doi.org/10.1103/PhysRevD.92.081301] 